Etude préliminaire portant sur le dépistage de la protéinurie à l'aide de bandelette urinaire chez les patients vivants avec le VIH au CHNU de Fann à Dakar.

\title{
Preliminary study on screening for proteinuria using urine test strips in patients living with HIV at Fann's CHNU in Dakar
}

Soumaré $\mathrm{M}^{1}$, Manga $\mathrm{N} \mathrm{M}^{2}$, Cissoko $\mathrm{Y}^{1}$, Fofana $\mathrm{A}^{1}$, Tine $\mathrm{J}^{2}$, Traoré $\mathrm{A}^{1}{ }^{1}$, Konaté $\mathrm{I}^{1}$, Dembélé $\mathrm{J}^{1}$, Dicko $\mathrm{H}^{3}$, Diakité $\mathrm{R}^{4}$, Dao $\mathrm{S}^{1}$, Diouf $\mathrm{B}^{5}$, Diop B $\mathrm{M}^{2}$.

1 : Service des Maladies Infectieuses, CHU du Point G, Bamako.

2 : Service des Maladies Infectieuses et tropical, CNHU Fann, Dakar.

3 : Service d'Anesthésie, Réanimation et bloc opératoire, CHU du Point G, Bamako.

4 : Service de Médecine nucléaire, CHU du Point G, Bamako.

5 : Service de Néphrologie et d'hémodialyse, CHU Aristide Le Dantec, Dakar.

Auteur correspondant : Dr Mariam Soumaré : mail:hammad276@yahoo.fr.

\section{Résumé :}

Les glomérulopathies et autres affections rénales sont fréquentes au cours de l'histoire naturelle du VIH même si leur incidence a diminué avec la trithérapie. Elles sont pratiquement toujours responsables d'une protéinurie et/ou d'une hématurie. Nous avons fait une étude préliminaire portant sur la prévalence de la protéinurie dépistée à l'aide de bandelettes urinaires chez les PVVIH suivies à la clinique des maladies infectieuses du CHNU de Fann à Dakar. Il s'agissait d'une étude prospective allant du $1^{\mathrm{er}}$ Août au 30 Octobre 2008. Ont été inclus de façon exhaustive les patients séropositifs pour le VIH à la fois vus en ambulatoire et en hospitalisation présentant une protéinurie sans infection urinaire intercurrente. Nous avons recueilli leurs données sociodémographiques, les éléments de l'histoire naturelle, les facteurs en rapport avec la protéinurie et la néphropathie. La saisie et l'analyse des données ont été faites avec le logiciel Epi Info version 6.04. Nous avons utilisé le test de Chi2 pour comparer les variables qualitatives et $\mathrm{p}<0,05$ a été retenu comme significative. Au total 100 patients ont été inclus, la moyenne d'âge

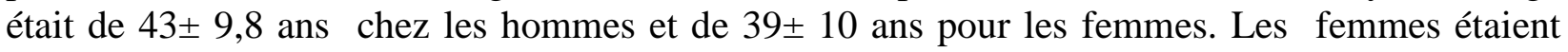
prédominantes (sex ratio $\mathrm{H} / \mathrm{F}=0,5$ ). Tous les patients étaient infectés par le VIH1, ils avaient été dépistés à un stade tardif d'immunodépression, la moitié était sous ARV avec le plus souvent le protocole AZT +3TC+ EFV. La prévalence de la protéinurie était de 47,9\%. Les facteurs significativement associés à la présence d'une protéinurie étaient: le sexe; le taux de CD4<350/. $\mathrm{mm}^{3}$; la charge virale $>100000$ copies $/ \mathrm{mm}^{3}$; l'IMC $<18,5$; le taux d'hémoglobine $<8,5$ $\mathrm{g} / \mathrm{dl}$, la chimioprophylaxie au cotrimoxazole et les stades cliniques OMS 3 et 4 . La fréquence de la néphropathie au cours du VIH au service des maladies infectieuses de Fann à Dakar suggère d'inclure son dépistage à la bandelette dans le bilan initial et de suivi de tout patient infecté par le VIH. Dans tous les cas il faudrait corriger tout facteur favorisant la survenue de cette néphropathie. La ponction biopsie rénale pourrait permettre des études plus approfondies.

Mots clés : protéinurie, insuffisance rénale, VIH, ARV, Dakar.

\begin{abstract}
:
Glomerulopathy and other kidney disorders are frequent during the natural history of HIV infection even if their incidence decreased with HAART. They are almost always responsible of proteinuria and/or hematuria. We have conducted a preliminary study on the prevalence of
\end{abstract}


proteinuria screened by urinary strip in PLWHIV followed up at the clinic of infectious diseases at Fann teaching hospital in Dakar. It was a prospective study from August $1^{\text {st }}$ to October $30^{\text {th }}$, 2008. We enrolled all patients both indoor and outdoor positive for HIV presenting a proteinuria without concurrent urinary infection. We collected sociodemographic data, natural history, factors related to proteinuria and nephropathy. Data entering and analysis were done using Epi Info version 6.04 software. We have used Chi square test to compare qualitative variables and $\mathrm{p}<0.05$ was set as significant threshold. A total of 100 patients were enrolled, the mean age was $43 \pm 9.8$ y/o in male and 39 \pm 10 y/o in female. Females were predominant (sex ratio M/F is 0.5). All patients were infected by HIV1, they have been diagnosed at a late stage of immunodeficiency, fifty percent was taking HAART, and the majority using AZT $+3 \mathrm{TC}+\mathrm{EFV}$ regimen. The prevalence of proteinuria was $47.0 \%$. Factors significantly associated with proteinuria were : the gender; CD4 count $<350 / \mathrm{mm}^{3}$; viral load $>100000$ copies $/ \mathrm{mm}^{3}$; BMI < $18.5 \mathrm{~kg} / \mathrm{m}^{2}$; Hemoglobin level $<8.5 \mathrm{~g} / \mathrm{dl}$, chemoprophylaxis with Cotrimoxazole and WHO clinical stage 3 and 4. The frequency of nephropathy during HIV infection in infectious diseases ward of Fann in Dakar suggest to include screening with urinary strip in the initial and follow up checking of all patient infected by HIV. And in all cases favoring factors of this nephropathy should be corrected. Kidney biopsy could help for better studies.

Key word: proteinuria, kidney failure, HIV, HAART, Dakar.

\section{Introduction}

Les glomérulopathies et autres affections rénales sont fréquentes au cours de l'histoire naturelle du VIH [1]. Les formes particulières de hyalinose segmentaire et focale, appelées "néphropathie associée au VIH" ou NVIH, ont été décrites pour la première fois en $1984 \mathrm{chez}$ les sujets de race noire infectés le plus souvent par le VIH1 $[2,3]$. La prévalence de la NVIH en Afrique chez les sujets non traités est estimée à $4 \%$ en 2006, soit environ 1 à 3 millions personnes [2]. Elle survient classiquement à la phase tardive de l'infection par le VIH. Elle se manifeste par un syndrome néphrotique d'installation brutale, avec une insuffisance rénale rapidement progressive. La protéinurie est le signe le plus précoce des glomérulopathies liées au VIH, d'où l'intérêt de sa recherche systématique dans le cadre du dépistage des néphropathies au cours du VIH. Cette méthode simple et accessible pourrait être d'un grand intérêt, surtout dans les pays à ressources limitées. Cependant la confirmation du diagnostic de néphropathie liée au VIH reste les résultats histochimiques réalisés par la ponction biopsie rénale, méthode invasive, qui doit être pratiquée selon certains auteurs, chez tout patient infecté par le VIH et présentant une protéinurie, même faible [2, 3]. Peu d'études ont été réalisées sur les néphropathies et VIH en Afrique [4-9]. Au Sénégal aucune étude n'a portée sur le dépistage de la protéinurie à la bandelette urinaire chez les PVVIH. Il nous a paru nécessaire d'étudier l'intérêt d'un dépistage systématique des néphropathies chez les PVVIH suivis à la clinique des Maladies Infectieuses du CHNU, afin de déterminer la fréquence des néphropathies et de décrire leur profil épidémiologique.

\section{Malades et méthode}

Nous avons mené une étude prospective à la clinique des maladies infectieuses du Centre Hospitalier National Universitaire de Fann du $1^{\text {er }}$ Août au 30 Octobre 2008. Ont été inclus les sujets âgés de 18 ans et plus, infectés par le VIH présentant une protéinurie positive ou non à l'examen aux bandelettes urinaires. Le refus de participer à l'étude, et la présence d'une infection urinaire évolutive constituait des critères de non inclusion, cependant les cas d'infection 
urinaire ont été inclus après traitement lorsque la protéinurie persistait. La collecte des données portait sur les données sociodémographiques, les antécédents, les aspects cliniques, biologiques et thérapeutiques. Le dépistage de la protéinurie était réalisée à l'aide de bandelettes urinaires (Combur $\underline{5}$-test $\left.{ }^{\circledR}\right)$. Ces bandelettes étaient plongées dans un échantillon d'urines fraîchement émises, puis la lecture était réalisée au bout d'une minute en s'aidant de l'abaque proposé par le fabriquant (Roche diagnostics $\mathrm{GmbH}$ ). Le seuil de détection des bandelettes était de 6 mg d'albumine par décilitre. Les résultats étaient reportés sous forme de croix allant de zéro (négatif) à 3 croix $(5 \mathrm{~g} / \mathrm{l})$ [10]. Un dépistage d'infection urinaire a été réalisé par bandelette sur la base de la présence de leucocytes avec ou sans nitrites dans les urines. La clairance de la créatinine était calculée en utilisant la formule de Cockcroft et Gault pour déterminer (le débit de la filtration glomérulaire et apprécier son stade) la présence d'une insuffisance rénale et apprécier sa gravité en fonction de la classification des insuffisances rénales chroniques [11]. La biopsie rénale n'a pas été faite, mais les patients qui présentaient une atteinte rénale sévère étaient envoyés en consultation spécialisée de néphrologie. Toutes ces données ont été saisies et analysées à l'aide de logiciel Epi Info version $6.04 \mathrm{du}$ CDC/OMS. Nous avons utilisé le test du chi2 pour la comparaison des variables qualitatives. Toute valeur de $\mathrm{p}<0,05$ était considérée comme significative.

\section{Résultats}

Un total de 100 patients ont été inclus, dont 67 suivis en ambulatoire et 33 en hospitalisation. Une prédominance féminine avec sex-ratio $(\mathrm{H} / \mathrm{F}) \quad 0,51$ a été notée. La tranche d'âge de 30-44 ans était la plus importante aussi bien chez les hommes que chez les femmes [Tableau I]. Cependant la moyenne d'âge était significativement plus élevée chez les hommes $43 \pm 9,8$ ans vs 39 \pm 10 ans chez les femmes. Les circonstances de découverte de l'infection par le VIH pour la plupart de nos patients étaient des symptômes évocateurs. Un antécédent de tuberculose été notée chez 25 de nos patients et celui de protéinurie gravidiques chez 6 patientes. Les affections chroniques sousjacentes étaient les cas d'HTA (6 cas), d'hémoglobinose AS (6 cas) et de diabète (2 cas). Une déshydratation a été retrouvée chez $26 \%$ de nos patients qui étaient tous des sujets hospitalisés. Nous avons retrouvés les œdèmes chez 5 patients et un cas d'HTA avec17/10 $\mathrm{cm} \mathrm{Hg}$ de tension chez qui la recherche de protéinurie à la bandelette était négative.

\section{Les infections opportunistes et stade clinique}

Les infections opportunistes en cours de traitement étaient les suivantes : tuberculose (22 patients), pneumocystose (3 patients), toxoplasmose cérébrale (2 patients) et cryptococcose neuroméningée (1patient). Les autres patients présentaient une diarrhée chronique et une fièvre au long court. La grande majorité des patients (83\%) étaient à un stade avancé de la maladie (stades III ou IV de l'OMS), [Tableau I]. Dans notre étude $47 \%$ des patients était à un stade sévère d'immunodépression avec un taux de CD4 $<200$ cellules $/ \mathrm{mm}^{3}$. Le taux moyen de lymphocytes CD4 chez nos patients était de $351 \pm 32$ cellules $/ \mathrm{mm}^{3}$. La charge virale réalisée dans les 6 derniers mois chez 30 patients, montrait que $53,3 \%$ avaient une charge virale supérieure à 100000 copie $/ \mathrm{mm}^{3}$. La majorité de nos patients avait un taux d'hémoglobine supérieur à $8,5 \mathrm{~g} / \mathrm{dl}$. Parmi les anomalies observées à l'examen aux bandelettes, la protéinurie prédominait $(47,9 \%)$ [Tableau III]. Une infection urinaire évolutive a été dépistée chez 25 patients avec la confirmation bactériologique dans deux cas par isolement d'une souche d'Escherichia coli et Staphylococcus saprophyticus. Seuls 5 patients présentaient une leucocyturie associée à une protéinurie. 
Ces patients ont été inclus après traitement et guérison de l'infection urinaire. La clairance était $\leq 60 \mathrm{ml} / \mathrm{min}$ dans $65,3 \%$ des cas, témoignant ainsi de l'existence d'une insuffisance rénale modérée et $44,3 \%$ étaient classés au stade 2 d'insuffisance rénale (MRC).

\section{Aspects thérapeutiques}

La moitié de nos patients était sous traitement antirétrovirale, les associations d'antirétroviraux les plus fréquemment utilisées étaient: AZT+3TC+EFZ (16\%); suivi de AZT+3TC+NVP (15\%). Le ténofovir a été utilisé chez 6 patients, tous présentant une protéinurie, il s'agissait d'un changement de l'AZT par le ténofovir pour cause d'anémie.

\section{Les facteurs associés à la présence d'une protéinurie}

Ils étaient, le stade OMS, le taux de CD4, le taux d'hémoglobine, la chimioprophylaxie au Cotrimoxazole et le traitement par les ARV [Tableau IV]. La protéinurie ne traduisait pas la gravité du degré d'insuffisance rénale. Les facteurs significativement associés à la gravité de MRC étaient l'âge $(\mathrm{p}=0,03), \quad$ l'IMC $(\mathrm{p}=0,002)$, le taux de CD4 ( $\mathrm{p}=0,03)$, le taux d'hémoglobine $(p=0,05)$ et le traitement ARV $(p=0,02)$. Les autres facteurs tels que le sexe, le stade clinique OMS, la protéinurie et l'hématurie n'étaient significativement corrélés au degré d'insuffisance rénal.

\section{Discussion}

\section{L'âge et le sexe}

La moyenne d'âge était de $43 \pm 9,8$ ans chez les hommes et $39 \pm 10$ ans chez les femmes. Ces données sont comparables à celles décrites dans les études antérieures réalisées dans le service par Mendy A et Diop SA [12, 13]. Les adultes jeunes constituent la population la plus touchée par l'infection par le VIH au Sénégal [14], en Afrique et dans le monde : Reid et al ont retrouvé une moyenne d'âge de 36,8 ans chez des patients originaire de l'Ouganda et du Zimbabwé [8], au Brésil Cavalcante et al ont eu une moyenne d'âge de $37 \pm 9$ ans [15]. La prédominance féminine (sex-ratio $\mathrm{H} / \mathrm{F}=0,5$ ) que nous avons notée, a été également retrouvée par N'Diaye et al au Sénégal avec un sex-ratio égal à 0,8 [16], ainsi que par Sow et al dans 4 pays africains (sexratio=0,6) [17]. En effet selon le rapport de l'ONUSIDA, en Afrique subsaharienne, pour dix hommes adultes vivant avec le $\mathrm{VIH}$, on compte quatorze femmes infectées [18].

\section{Les aspects cliniques}

La majorité de nos patients $(77 \%)$ ont été dépistés à un stade symptomatique. Mendy A [12] a souligné aussi cette notion, avec un retard important au diagnostic de la maladie chez les patients admis au service des Maladies infectieuses de Fann. Ce retard constaté par beaucoup d'auteurs africains est lié à l'insuffisance des connaissances des populations sur le VIH/Sida et aux nombreuses occasions manquées lors des consultations antérieures [13]. La maladie a été découverte lors d'un dépistage systématique chez 23 patients dont 18 femmes dans notre série. En effet, les femmes ont plus d'opportunités de dépistage lors des bilans de la grossesse.

Concernant les antécédents, la protéinurie a été retrouvée chez 6 patientes, c'était en fait des cas de protéinuries gravidiques survenu au moment de la grossesse. Les antécédents en rapport avec le $\mathrm{VIH}$ chez nos patients étaient dominés par les diarrhées chroniques $(39 \%)$ et la tuberculose $(25 \%)$, le même constat a été fait par Mendy [12] et Diakhaté [19], au cours de travaux menées dans le service. La tuberculose reste la principale infection opportuniste retrouvée dans l'histoire de l'infection à VIH des patients en Afrique sub-saharienne [5].

\section{Les aspects paracliniques}

Dans notre étude, $47 \%$ des patients avaient un taux de CD4<200 cellules $/ \mathrm{mm}^{3}$. Le taux moyen de CD4 de 351 cellules $/ \mathrm{mm}^{3}$ était comparable à celui de 363 cellules $/ \mathrm{mm}^{3}$ retrouvé au Brésil par Cavalcante [15]. Chez 
les 30 patients qui avaient bénéficié d'un dosage de la charge virale, 53,3\% avaient une charge virale supérieure à 100000 copies, ceci diffère des $37 \%$ retrouvé au Brésil en 2004 [15]. Dans notre étude, la prévalence de la protéinurie était de $47,9 \%$. Ce taux est plus proche des $38 \%$ décrit par Emem et al au Nigeria [4], il est cependant nettement plus élevé que les taux retrouvé dans d'autres études au niveau mondial. En effet aux USA, Kimmel et al [23] en 1993 trouvaient un taux de $29,8 \%$, qui était supérieur à ceux décrits par Crowley et al [1] en 2001 et Gupta et al [20] en 2007 qui étaient respectivement de $14 \%$ et $10 \%$. Une étude réalisée au Brésil en 2004 par Cavalcante et al avait trouvé une prévalence de 5,6\% [15]. La grande disparité dans les résultats obtenus par ces auteurs s'explique en partie par les différences entre les populations étudiées. En effet au sein de ces populations la protéinurie était plus fréquente chez les sujets de race noire. La proportion de sujets sous trithérapie pourrait aussi être un élément explicatif de cette différence. Wools-Kaloustian et al ont mis en évidence une protéinurie chez 5,9\% de patients naïs de tout traitement ARV au Kenya [9]. Cependant d'autres facteurs liés à l'environnement, au mode de vie et à la présence d'autres causes de protéinurie (Diabète, maladies de système...), pourraient aussi être pris en compte. La clairance de la créatinine se situait entre $60-89 \mathrm{ml} / \mathrm{mn}$ chez $47,4 \%$ des cas, taux comparable aux $45 \%$ décrits par Reid et al [8] en Ouganda et au Zimbabwé. Au Kenya Wools-Kaloustian [9] et Mulenga en Zambie [6] ont trouvé respectivement une clairance $<60 \mathrm{ml} / \mathrm{mn}$ chez $13 \%$ et $26,5 \%$ des patients, taux inférieur au notre qui était de $35,8 \%$. Nous n'avons pas utilisé la Modification of Diet on Renal Disease Formulae (MDRF), proposée par certains auteurs comme plus adaptée pour les sujets de race noire avec un facteur de correction de 1,21, car Stöhr et al ne l'ont pas trouvé supérieure à la formule de
Coccroft et Gault classique dans les travaux qu'ils ont réalisé chez des sujets d'origine africaine infectés par le VIH [21]. Les patients qui présentaient des signes d'atteinte rénale (protéinurie +/- insuffisance rénale modérée ou sévère) ont été adressés en consultation de néphrologie pour de plus amples investigations (biopsie rénale) et un meilleur suivi. En Afrique du Sud, une étude a retrouvé $85,7 \%$ de HIV Associated Nephropathy (HIVAN) à la biopsie chez un groupe de patients qui présentaient une protéinurie persistante et $72,4 \%$ chez ceux souffrant de néphropathie chronique [7]. Une étude récente réalisée à Dakar avec réalisation de biopsies rénale chez des patients souffrant d'une néphropathie chronique a retrouvé $7,1 \%$ de lésions glomérulaires segmentaires et focales (FSGS) avec «collapsing », similaires à celles décrites dans le cadre du HIVAN [22]. Il serait pertinent après notre étude pilote de prévoir des études plus poussées avec un dosage quantitatif de la protéinurie de 24 heures car il peut y avoir des faux positifs ou négatifs à la bandelette.

\section{Aspects analytiques}

Les facteurs en rapport avec la protéinurie

La protéinurie était significativement plus fréquente chez les hommes $(61,7 \%$ vs $37,8 \%, \mathrm{p}=0,02)$. Une étude faite au Brésil en 2004 sur la protéinurie et le VIH trouve le même résultat (70\%) [15].

L'âge avancé était un facteur associé à la protéinurie chez les PVVIH au Nigéria [4]. Nous avons trouvé une fréquence des protéinuries plus élevée chez les patients de 50 ans et plus (52,7 vs 42,1 ; NS). Un IMC< 18,5; un taux de $\mathrm{CD} 4<350$ et un taux $\mathrm{d}^{\prime} \mathrm{Hb}<8,5 \mathrm{~g} / \mathrm{dl}$, ont été significativement associées à une protéinurie chez nos patients. Des résultats similaires ont été trouvés dans plusieurs études en Afrique, qui concluent que la dénutrition et l'anémie constituent des facteurs de risque de protéinurie $[23,8]$. Plusieurs auteurs ont noté la relation qui 
existe entre un taux de CD4 bas et la survenue d'une protéinurie: Cavalcante $\mathrm{A}$ et al au Brésil ont noté une fréquence significativement plus élevée $(p<0,04)$ d'une protéinurie persistante chez les PVVIH avec un taux de CD4<200 [15] ; le même constat a été fait par Crowley et al [1]. Il est aujourd'hui établi qu'une immunodépression majeure avec un taux de CD4 bas constitue un facteur de risque de survenue d'une HIVAN [22-24], c'est ce qui explique la régression de la protéinurie sous traitement ARV avec restauration immunitaire. Dans notre série, la protéinurie est significativement plus fréquente chez les sujets non traités. S'agissant de la charge virale et la protéinurie : parmi les 30 patients qui avaient fait la charge virale, elle était détectable chez 27 patients et indétectable chez seulement 3 patients. Chez ceux qui avaient une charge virale détectable, elle était supérieure à $100000 \mathrm{chez} 16$ patients et tous avaient une protéinurie. Plusieurs auteurs ont fait la relation entre charge virale supérieure à 100000 copies $/ \mathrm{mm}^{3}$ et la protéinurie [1,15]. Au Kenya, WoolsKaloustian et al ont noté que la survenue d'une protéinurie était associée à un faible poids, un taux de CD4 bas, un taux d'hémoglobine bas, un antécédent de tuberculose et la présence d'une insuffisance rénale [9].

\section{Les facteurs associés à l'insuffisance rénale}

Les facteurs significativement liés à une souffrance rénale modérée à sévère dans notre série ont été : âge $\geq 50$ ans, une dénutrition (IMC $\leq 18,5)$, anémie $(\mathrm{Hb}<$ $8,5 \mathrm{~g} / \mathrm{dl})$, taux de CD4 $<350 / \mathrm{mm}^{3}$ et l'absence de traitement ARV en cours. Une étude récente réalisée au Nigéria a noté comme nous, une relation entre un âge avancé (>60 ans), un IMC corporel bas et un taux de CD4 bas et la survenue d'une souffrance rénale [4]. Les auteurs de ce travail ont également noté qu'un taux de cholestérol bas et une hypoalbuminémie étaient aussi associés à une néphropathie. $\mathrm{Au}$ Kenya, Wools-Kaloustian et al [9] ont noté entre les patients présentant une Clcréat $<60 \mathrm{ml} / \mathrm{mn}$ et ceux avec une Clcréat $\geq$ $60 \mathrm{ml} / \mathrm{mn}$, une différence significative en terme de : pression artérielle moyenne, taux de CD4 moyen, taux d'hémoglobine moyen, fréquence d'une protéinurie, fréquence d'une maigreur. En analyse multivariée, seule la maigreur et l'anémie sont restée significativement associées à une baisse de la clairance de la créatinine. Nous n'avons pas trouvé cependant dans notre étude, une relation significative entre protéinurie et insuffisance rénale modérée à sévère. Cependant, ces deux marqueurs de souffrance rénale sont moins importants chez nos malades sous traitement antirétroviral. La régression de la souffrance rénale chez les patients sous traitement antirétroviral a été constatée par plusieurs auteurs à travers le monde, même pour les protocoles comportant des médicaments potentiellement néphrotoxiques comme le Ténofovir [23-25]. La mise en évidence d'une souffrance rénale au moment du dépistage de l'infection par le VIH est de plus en plus considérée comme critère de mise sous traitement antirétroviral [1]. Nous ne nous sommes pas intéressés au devenir des patients qui avaient une souffrance rénale sévère, mais une étude réalisée en Zambie a noté une surmortalité chez les patients ayant une insuffisance rénale modérée à sévère à l'initiation du traitement ARV, après un suivi de 90 jours

\section{CONCLUSION :}

Les glomérulopathies et autres affections rénales sont fréquentes au cours de l'histoire naturelle du VIH. Dans notre étude, il ressort que le taux de CD4 bas et la charge virale élevée sont les principales causes de protéinurie chez les PVVIH d'où l'intérêt d'une prise en charge précoce des patients. La prise en charge de la dénutrition et de l'anémie pourrait constituer des stratégies de 
prévention de la survenue d'une insuffisance rénale.

\section{References}

1. Crowley ST, Cantwell B, Abu-Alfa A et al. Prevalence of persistent asymptomatic proteinuria in HIV-infected outpatients and lack of correlation with viral load. Clin Nephrol 2001;55:1-6.

2. Weiner NJ, Goodman JW, Kimmel PL. The HIV associated renal diseases: current insight into pathogenesis and treatment. Kidney Int 2003; 63: 1618-31.

3. Tourret J, Tostivint I, Deray G. Manifestations néphrologiques. VIH, Edition Doin Paris, 2007; 231-43.

4. Emem CP, Arogundade F, Sanusi A, Adelusola K, Wokoma F, Akinsola A. Renal disease in HIV-seropositive patients in Nigeria: an assessment of prevalence, clinical features and risk factors. Nephrol Dial Transplant. 2008; 23(2):741-6.

5. Garcia JM, Gowns E, Ghys PD. National population based HIV prevalence surveys in sub-Sahara Africa: results and and implication for HIV and AIDS estimates. Sex transm Infected 2006; 82: 64-70.

6. Mulenga LB, Kruse G, Lakhi S, Cantrell RA, Reid SE, Zulu I et al. Baseline renal insufficiency and risk of death among HIV-infected adults on antiretroviral therapy in Lusaka, Zambie. AIDS 2008;14:1821-7.

7. Han TM, Nicker S, Ramdial PK et al. A cross selectionnal study of HIV seropositive patients with varying degrees of proteinuria in South Africa. Kidney Int 2006;69:2243-50.

8. Peter PJ, Moore D.M., Meronin J. et AL. Antiretroviral therapy improves renal function among HIV infected Ugandans. Kidney Int, 2008;74:845-7.

9. Wools-Kaloustian K., Gupta SK., Muloma E., Owino-Ong'or W., Sidle J., Aubrey RW., Shen J., Kipruto K., Zwickl
BE. and Goldman M. Renal disease in an antiretroviral-naïve HIV-infected outpatient population in Western Kenya. Nephrol Dial Transplant 2007;22: 220812.

10. Bandelette urinaire MULTISTIX Bayer corporation. réf. 091s9477 12289, Bayer diagnostics, Tour Horizon, 52, quai de Dion Boulon, 92807 puteaux cedex bayer (suisse).

11. Froissart M, Delanaye P, Seronie V, Cistol JP. Evaluation of the renal function: an update. Ann Biol Clin 2008; 66 (3): 269-75.

12. Mendy A. Itinéraires thérapeutiques avant la découverte de la séropositivité et facteurs du dépistage tardif de l'infection à VIH. Thèse de Med, Dakar 2007 N$^{\circ} 8$.

13. Diop SA. Caractéristiques et devenir des patients vivant avec le VIH perdus de vue au service des maladies infectieuses de FANN. Mémoire CES maladies infectieuses, Dakar $2008 \mathrm{~N}^{\circ} 108$.

14. Enquête démographique et de santé : Sénégal 2005, Centre de Recherche pour le développement Humain/ Ministère de santé et de la Prévention Médicale, ORC, Macro 2006. p 17-8.

15. Mattos Cavalcante MAG, Coelho SN, Lacerda HR. Prevalence of persistent proteinuria in Stable HIV/AIDS Patients and its association with HIV Nephropathy. Braz J of Infect Dis 2007; 11(5): 456-61.

16. Ndiaye J. Effets secondaires de la thérapie antirétrovirale dans la prise en charge des patients vivants avec le VIH/SIDA.Thèse de Med, Dakar 2004 ; $\mathrm{N}^{\circ} 41$.

17. Sow PS, Otieno LF, Bissagnene E, Kityo C, Bennink R, Clevenbergh P, Wit FW, Waalberg E, Lange JM. Cohort program to Evaluate Access to Antiretroviral therapy and Education project Team. J. Acquis Immune Defic. Syndr. 2007 Mar 1; 44 (3): 262-7. 
18. ONUSIDA: Le point sur l'épidémie de sida 2007. data.Unaids.org/pub/Epislides/2007/2007 epiupdate_fr.pdf. Consultation du 15/07/08

19. Diakhate N. Morbidité et mortalité hospitalière du SIDA dans un service de pathologie infectieuse à Dakar: bilan de 12 ans d'activité. Thèse de Med, Dakar, $1998 ; \mathrm{N}^{\circ} 05$.

20. Gupta SK et al. The effects of highly active antiretroviral therapy on albuminuria in HIV-infected persons: results from a randomised trial. Nephrol Dial Transplant. 2005; 20(10):2237-42.

21. Stohr W, Walker AS, Munderi P, Tugume S, Gilks CF, Darbyshire JH, Hakim J, Dart T. Estimating glomerular filtration rate in $\mathrm{HIV}$-infected adults in Africa: comparaison of Cockroft-Gault and modification of Diet in Renal Disease formulate. Antivir Ther 2008;13(6): 76170 .

22. Niang A, Dial C, Ka EF, Leye A, Pouye A, Ka MM et al. Nephrotic syndrom with focal and segmental glomerulosclerosis in Dakar : epidemiological and clinicopathological characteristics (about 134 cases). Dakar Med 2008; 53:45-51.

23. Gerntholtz TE, Goetsch SJ, Katz I. HIV-related nephropathy: a South Africa perspective. Kidney Int 2006; 69: 188591.

24. Lucas GM, Eustace JA, Sozio S et al. Highly active antiretroviral therapy and the incidence of HIV-1 associated nephropathy: a 12 year cohort study. AIDS 2004; 18: 541-6.

25. Kimmel PL, Umana WO, Bosh JP et al. Abnormal urinary protein in HIVinfected patients. Clin Nephrol 1993 ; 39 : $17-21$. 


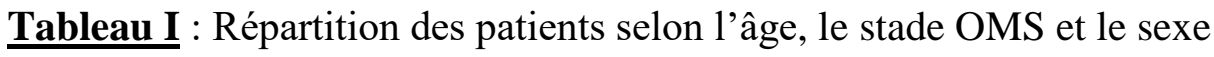

\begin{tabular}{ccccc} 
& & Féminin & Masculin & Total \\
\hline \multirow{2}{*}{ Tranche d'âge } & $\mathbf{3 0}-\mathbf{4 4}$ & 36 & 1 & 9 \\
(années) & $\mathbf{4 5}-\mathbf{5 9}$ & 20 & 19 & 55 \\
& $\mathbf{6 0}-\mathbf{7 4}$ & 2 & 12 & 32 \\
& I & 8 & 2 & 4 \\
\hline \multirow{2}{*}{ Stade OMS } & II & 7 & 1 & 9 \\
& III & 39 & 1 & 8 \\
& IV & 12 & 18 & 57 \\
\hline Total & & 66 & 34 & 26 \\
\hline
\end{tabular}

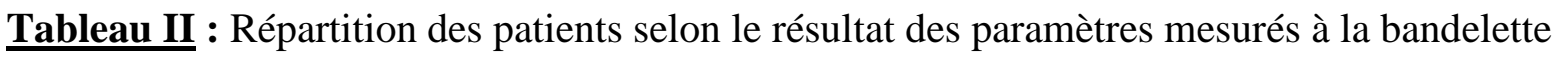
urinaire

\begin{tabular}{|c|c|c|c|c|c|}
\hline \multirow{2}{*}{$\begin{array}{ll}\text { Résultat } & \text { Paramètres }\end{array}$} & \multicolumn{2}{|c|}{ Positif } & \multicolumn{2}{|c|}{ Négatif } & \multirow[t]{2}{*}{ Total } \\
\hline & $N$ & $\%$ & $N$ & $\%$ & \\
\hline Protéinurie & 46 & 46,0 & 54 & 54,0 & \\
\hline Hématurie & 17 & 17,0 & 83 & 83,0 & $100=100 \%$ \\
\hline Leucocyturie & 25 & 25,0 & 75 & 75,0 & \\
\hline Nitrite & 8 & 8,0 & 92 & 92,0 & \\
\hline
\end{tabular}




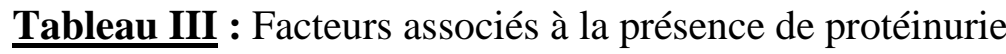

\begin{tabular}{|c|c|c|c|c|}
\hline \multirow[t]{2}{*}{ Paramètres } & \multicolumn{2}{|c|}{ Protéinurie } & \multirow[t]{2}{*}{ Total (n) } & \multirow[t]{2}{*}{$\mathbf{p}$} \\
\hline & Oui & Non & & \\
\hline \multicolumn{5}{|l|}{ Sexe } \\
\hline M & 21 & 13 & 34 & 0,02 \\
\hline $\mathrm{F}$ & 25 & 41 & 66 & \\
\hline \multicolumn{5}{|c|}{ Tranche d'âge (ans) } \\
\hline $15-44$ & 27 & 37 & 64 & 0,30 \\
\hline$\geq 45$ & 19 & 17 & 36 & \\
\hline \multicolumn{5}{|l|}{ IMC $\left(\mathrm{kg} / \mathrm{m}^{2}\right)$} \\
\hline$\leq 18,5$ & 30 & 13 & 43 & 0,00003 \\
\hline$>18,5$ & 16 & 41 & 57 & \\
\hline \multicolumn{5}{|l|}{ Stades OMS } \\
\hline $1-2$ & 2 & 15 & 17 & 0,004 \\
\hline $3-4$ & 41 & 42 & 83 & \\
\hline \multicolumn{5}{|l|}{ TCD4 $\left(\right.$ cell $\left./ \mathrm{mm}^{3}\right)$} \\
\hline$<350$ & 43 & 20 & 63 & 0,0002 \\
\hline$\geq 350$ & 7 & 20 & 27 & \\
\hline \multicolumn{5}{|l|}{ CV (copies/ml) } \\
\hline$<50$ & 0 & 3 & 3 & 0,05 \\
\hline$\geq 50$ & 16 & 11 & 27 & \\
\hline \multicolumn{5}{|l|}{ Taux d'Hb (g/dl) } \\
\hline$<8,5$ & 22 & 9 & 31 & 0,0007 \\
\hline$\geq 8,5$ & 24 & 45 & 69 & \\
\hline \multicolumn{5}{|l|}{ Sévérité IRC } \\
\hline Stade $1-2$ & 26 & 35 & 61 & 0,2 \\
\hline Stade 3-4 & 19 & 15 & 34 & \\
\hline \multicolumn{5}{|l|}{ Hématurie } \\
\hline Oui & 9 & 8 & 17 & 0,5 \\
\hline Non & 37 & 46 & 83 & \\
\hline \multicolumn{5}{|l|}{$\begin{array}{l}\text { Prophylaxie au } \\
\text { cotrimoxazole }\end{array}$} \\
\hline Oui & 40 & 6 & 46 & 0,00017 \\
\hline Non & 28 & 26 & 54 & \\
\hline \multicolumn{5}{|l|}{ ARV } \\
\hline Oui & 17 & 33 & 50 & 0,016 \\
\hline Non & 29 & 21 & 50 & \\
\hline
\end{tabular}

